(C) 1998 International Press

Adv. Theor. Math. Phys. 2 (1998) 1373-1404

\title{
Type IIA D-Branes, K-Theory and Matrix Theory
}

Petr Hořava ${ }^{a}$

${ }^{a}$ California Institute of Technology,

Pasadena,

CA 91125, USA

horava@theory.caltech.edu

\begin{abstract}
We show that all supersymmetric Type IIA D-branes can be constructed as bound states of a certain number of unstable non-supersymmetric Type IIA D9-branes. This string-theoretical construction demonstrates that D-brane charges in Type IIA theory on spacetime manifold $X$ are classified by the higher $\mathrm{K}$-theory group $\mathrm{K}^{-1}(X)$, as suggested recently by Witten. In particular, the system of $N$ D0-branes can be obtained, for any $N$, in terms of sixteen Type IIA D9-branes. This suggests that the dynamics of Matrix theory is contained in the physics of magnetic vortices on the worldvolume of sixteen unstable D9-branes, described at low energies by a $U(16)$ gauge theory.
\end{abstract}

e-print archive: http://xxx.lanl.gov/abs/hep-th/9812135 


\section{Introduction}

When we consider individual D-branes in Type IIA or Type IIB string theory on $\mathbf{R}^{10}$, we usually require that the branes preserve half of the original supersymmetry, and that they carry one unit of the corresponding RR charge. These requirements limit the D-brane spectrum to $p$-branes with all even values of $p$ in Type IIA theory, and odd values of $p$ in Type IIB theory.

Once we relax these requirements, however, we can consider $\mathrm{D} p$-branes with all values of $p$. In Type IIA theory, we can consider $p$-branes with $p$ odd, and in particular, a spacetime-filling 9-brane. All these states are nonsupersymmetric unstable excitations in the corresponding supersymmetric string theory. Indeed, there is always a tachyon in the spectrum of the open string connecting one such Type IIA $(2 p-1)$-brane to itself. Thus, such D-brane configurations (and their counterparts on spacetimes of non-trivial topology) are highly unstable, and one expects that they should rapidly decay to the supersymmetric vacuum, by a process that involves tachyon condensation on the worldvolume. This is of course in perfect agreement with the field content of the corresponding low-energy supergravity in spacetime - there are no RR fields that could couple to any conserved charges carried by such non-supersymmetric branes.

This does not seem to leave much room for surprises, but in fact, the full story is much more interesting. Configurations of unstable D-branes can sometimes carry lower-dimensional D-brane charges, and therefore, when the tachyon rolls down to the minimum of its potential and the state decays, it can leave behind a supersymmetric state that differs from the vacuum by a lower-dimensional D-brane charge - in other words, the state decays into a supersymmetric D-brane configuration. Typically, one can then represent the supersymmetric D-brane state as a bound state of the original system of unstable D-branes.

This setup generalizes a special case studied in $[1,2]$, where one starts with an unstable configuration of an equal number of stable $p$-branes and stable anti- $p$-branes (or $\bar{p}$-branes for short), and finds lower-dimensional stable D-brane states as bound states in this system. The $p$-brane $\bar{p}$-brane system is unstable, and the instability manifests itself by the presence of a tachyon in the spectrum of the $p-\bar{p}$ open strings at brane separations shorter than the string scale [3]-[7]. In Type IIB or Type I string theory, one can use this construction to represent any stable D-brane state as a bound state of a certain number of 9-brane $\overline{9}$-brane pairs $[1,2]$.

In some cases, the unstable non-supersymmetric state decays into a stable 
state that is not supersymmetric, but is protected from further decay by charge conservation. One typical example of such states is the $S O(32)$ spinor of Type I theory [1], which is non-supersymmetric but stable, since it is the lowest spinor state in the theory. Such non-supersymmetric D-branes can be found using a direct boundary state construction [8] , or alternatively as bound states of $p$-brane $\bar{p}$-brane pairs [1] . This remarkable bound-state construction was discovered by Sen [1] (following some earlier work in [9] ), and was further systematized by Witten [2] . It turns out that the proper setting for all conserved D-brane charges in general compactifications is, in fact, K-theory $[10,2]$. (Some early indications of possible connections between $\mathrm{D}$-branes and K-theory can be found in [11]-[18].)

In this paper, we generalize the construction that uses unstable configurations of pairs of stable branes, and consider bound states in general unstable non-supersymmetric configurations of D-branes such as the unstable 9-brane discussed above, where the individual D-branes are no longer required to be stable.

Our motivation for this generalization will be clear from the following example. We will be interested in stable D-branes in Type IIA string theory, for simplicity in $\mathbf{R}^{10}$. In Type IIB theory, we can in principle construct any stable $\mathrm{D}$-brane state as a bound state of a certain number of 9-brane $\overline{9}$-brane pairs wrapping the whole spacetime. It is certainly desirable to have, on the Type IIA side, a similar construction that would enable us to study stable D-branes as bound states in unstable configurations of branes of maximal dimension. However, there are no stable D9-branes in Type IIA string theory! While we can indeed represent any stable D-brane of Type IIA as a bound state of an 8-brane $\overline{8}$-brane system, this construction requires a preferred choice of a submanifold of codimension one in space-time, representing the worldvolume of the 8-brane $\overline{8}$-brane system. Therefore, it breaks some of the spacetime symmetries that we want to keep manifest in the theory, and limits the kinematics of branes that can be studied this way. One of the main points of this paper is to present a string-theoretical construction that keeps all spacetime symmetries manifest. This construction enables us to consider any stable D-brane of Type IIA theory as a bound state of a system of the unstable 9-branes discussed above. In fact, this construction turns out to be intimately related to the statement that D-brane charges in Type IIA string theory are classified by the higher $\mathrm{K}$-theory group $\mathrm{K}^{-1}(X)$ of the spacetime manifold $X$, suggested recently by Witten in [2] .

This paper is organized as follows.

In section 2 we briefly review the relation of Type IIB D-brane charges 
and bound-state constructions to K-theory, and preview the Type IIA case.

In section 3.1 we introduce the unstable 9-brane of Type IIA string theory. General 9-brane configurations wrapping spacetime manifold $X$ are studied in section 3.2. We argue that inequivalent configurations of 9-branes - modulo 9-branes that can be created from or annihilated to the vacuum - are classified by the higher $\mathrm{K}$-theory group $\mathrm{K}^{-1}(X)$. In section 3.3 we show that any given stable D-brane configuration of Type IIA string theory can be represented as a bound state of a certain number of unstable Type IIA 9-branes. In the worldvolume of the 9-branes, the bound state appears as a stable vortex in the tachyon field, accompanied by a non-trivial gauge field carrying a generalized magnetic charge. In the particular case of bound states in codimension three, this precisely corresponds to the 't HooftPolyakov magnetic monopole. We generalize our discussion to the case of Type $\mathrm{I}^{\prime}$ theory in section 3.4 , and argue that Type $\mathrm{I}^{\prime} \mathrm{D}$-brane charges are similarly classified by the Real $\mathrm{K}$-theory group $\mathrm{KR}^{-1}(X)$.

In section 4 we focus on possible implications of our construction to Matrix theory [19]. We use our 9-brane bound-state construction to study a general system of $N$ D0-branes in Type IIA theory. First we show that a D0-brane can be constructed as a bound state of sixteen unstable D9branes. The low-energy worldvolume theory on the 9-branes is a certain non-supersymmetric $U(16)$ gauge theory, with a tachyon in the adjoint representation of $U(16)$. In this worldvolume theory, the D0-brane is represented as a topologically stable vortex-monopole configuration of the tachyon and the gauge field. Multiple D0-brane configurations are in general also described by sixteen 9-branes, and appear as multi-vortex configurations in the $U(16)$ gauge theory on the spacetime-filling worldvolume. This construction thus leads to the intriguing possibility that the dynamics of Matrix theory as described by a particular limit of the system of $N$ D0-branes of Type IIA string theory - can be contained in the dynamics of vortices on the worldvolume of a fixed system of sixteen D9-branes, with the individual D0-branes represented by vortices in the worldvolume field theory. This construction exhibits some striking similarities with the holographic field theory of [20] .

While this paper was being finished, another paper appeared [21] whose section 6 partially overlaps with some parts of our section 3.3. 


\section{K-Theory and Type IIA D-Branes}

In this section we first review some highlights of [2] (mostly in the context of Type IIB theory), which will give us the opportunity to present some background on K-theory [22]-[26] that will be useful later in the paper. In section 2.2 we set the stage for our further discussion of D-brane charges and bound states in Type IIA theory.

\subsection{Type IIB on $X$ and $\mathrm{K}(X)$}

Consider supersymmetric $p$-branes and $\bar{p}$-branes of Type IIB (or Type IIA) string theory, wrapped on a spacetime manifold $X$ of dimension $p$. The lowest states of the open strings connecting $N$ such $p$-branes give rise to a worldvolume $U(N)$ gauge field on a Chan-Paton bundle $E$ of complex dimension $N$. Similarly, $N^{\prime} \bar{p}$-branes will carry a Chan-Paton bundle $E^{\prime}$ of dimension $N^{\prime}$ and a $U\left(N^{\prime}\right)$ gauge field. The open string connecting a $p$-brane to a $\bar{p}$-brane will have the opposite GSO projection, and its lowest mode will be a tachyon field $T$ in $\left(N, \overline{N^{\prime}}\right)$ of the gauge group $U(N) \times U\left(N^{\prime}\right)$.

The D-brane charge of the configuration will be preserved in processes where $p$-brane $\bar{p}$-brane pairs are created from or annihilated to the vacuum. Configurations that can be created or annihilated correspond to $N p$-branes with bundle $F$ and $N \bar{p}$-branes with a bundle $F^{\prime}$ that is topologically equivalent to $F$. Thus, invariant $D$-brane charges correspond to equivalence classes of pairs of bundles $\left(E, E^{\prime}\right)$, where two pairs $\left(E_{1}, E_{1}^{\prime}\right)$ and $\left(E_{2}, E_{2}^{\prime}\right)$ are equivalent if $\left(E_{1} \oplus F, E_{1}^{\prime} \oplus F\right)$ is isomorphic to $\left(E_{2} \oplus G, E_{2}^{\prime} \oplus G\right)$ for some $F$ and $G$. (If $F$ corresponds to brane antibrane pairs being created from the vacuum, $G$ corresponds to pairs annihilated to the vacuum.) The set $\mathrm{K}(X)$ of such equivalence classes of pairs of bundles on $X$ forms a group, called the $\mathrm{K}$-theory group of $X$. The image of $(E, 0)$ in $\mathrm{K}(X)$ is sometimes denoted by $[E]$. Each element in $\mathrm{K}(X)$ can be written as $[E]-\left[E^{\prime}\right]$ for some bundles $E$ and $E^{\prime}$.

Consider configurations of 9-branes and $\overline{9}$-branes in Type IIB string theory, wrapping a spacetime manifold $X$. Tadpole cancellation requires an equal number of branes and antibranes. In K-theory, one can show - for $X$ a connected compact manifold - that $\mathrm{K}(X)$ canonically factorizes as $\widetilde{\mathrm{K}}(X) \oplus \mathbf{Z}$, where the integer in $\mathbf{Z}$ is simply $N-N^{\prime}$, the difference between the number of $p$-branes and the number of $\bar{p}$-branes. For a non-compact manifold $Y$, one defines $\mathrm{K}(Y)=\widetilde{\mathrm{K}}(\tilde{Y})$, where $\tilde{Y}$ is a compactification of $Y$ by adding a point at infinity. Thus, on a general spacetime manifold $X, \mathrm{D}$-brane charges 
of tadpole cancelling Type IIB 9-brane $\overline{9}$-brane configurations are classified by the reduced $\mathrm{K}$-theory group $\widetilde{\mathrm{K}}(X)$.

Each class in $\widetilde{\mathrm{K}}(X)$ is represented by a system with an equal number of 9-branes and $\overline{9}$-branes wrapping $X$, with the class in $\mathrm{K}(X)$ given by the difference of the Chan-Paton bundles on 9-branes and $\overline{9}$-branes, $[E]-$ $\left[E^{\prime}\right]$. Open strings ending on all possible pairs of these branes will give rise to a $U(N) \times U(N)$ gauge field, and the tachyon field $T$ in the $(N, \bar{N})$ representation of the gauge group. Together, these bosonic fields form an object

$$
\left(\begin{array}{ll}
A & T \\
\bar{T} & A^{\prime}
\end{array}\right)
$$

known in the mathematical literature as a "superconnection" on $X$ [27] .

The tachyon has the tendency to roll down to the minimum of its potential located at some $T=T_{0}$. We do not know the exact form of the tachyon potential, but it was argued in [2] that at the minimum of the potential, all eigenvalues of $T_{0}$ are equal, and therefore $T_{0}$ breaks the gauge symmetry from $U(N) \times U(N)$ to the diagonal $U(N)$.

Any lower-dimensional stable D-brane of Type IIB theory, wrapping a submanifold $Y$ in spacetime, can be constructed as a bound state in a system of $N$ 9-branes and $N \overline{9}$-branes, which locally near $Y$ looks like a topologically stable vortex of the tachyon field. ${ }^{1}$ This can be seen as follows. Stable values of $T_{0}$ correspond to the vacuum manifold

$$
\mathcal{V}_{0}(N)=(U(N) \times U(N)) / U(N),
$$

which is topologically equivalent to $U(N)$. Thus, the tachyon will support stable defects in codimension $2 k$, classified by the non-zero homotopy groups of the vacuum manifold, $\pi_{2 k-1}\left(\mathcal{V}_{0}(N)\right)=\mathbf{Z}$ (for stable values of $N$ ). In order for these defects to carry finite energy, the vortex of winding number $n$ in the tachyon field must be accompanied by a non-trivial gauge field configuration carrying $n$ units of the corresponding topological charge. In the simplest case of one $p$-brane $\bar{p}$-brane pair, a vortex of codimension two with vorticity $n$ carries $n$ units of the magnetic flux in one of the $U(1)$ groups.

The core of the vortex lies along a submanifold $Y$ of codimension $2 k$ in $X$. Outside a small core around the submanifold $Y$ in $X$, the tachyon condenses to its vacuum value. It is believed $[1,9]$ that the negative energy density corresponding to the vacuum condensate of the tachyon field is equal

\footnotetext{
${ }^{1}$ For the purposes of this paper, it will be sufficient to consider only branes stretching along submanifolds $\mathbf{R}^{m}$ of the flat spacetime $\mathbf{R}^{10}$. The general case of Type IIB D-branes wrapping general submanifolds $Y$ in general spacetime $X$ is discussed in [2] .
} 
in magnitude to the positive energy density due to the non-zero tension of the $p$-brane $\bar{p}$-brane system wrapping $X$. Consequently, the total energy density away from the core of the bound state rapidly approaches zero, and the configuration is very close to the supersymmetric vacuum. Thus, the process of tachyon condensation leaves behind an object wrapped on $Y$ that carries the charge of a supersymmetric D-brane wrapping $Y[1]$.

In terms of $\mathrm{K}$-theory, this construction corresponds to the embedding of a non-trivial class in $\mathrm{K}(Y)$ - describing a D-brane wrapping $Y$ - into $\mathrm{K}(X)$, where it corresponds to the bound state of a 9 -brane $\overline{9}$-brane configuration wrapping $X$. This embedding is realized by a classic $\mathrm{K}$-theory construction [25], which selects - for a $Y$ of codimension $2 k$ in $X$ - a preferred value of the number of 9-brane $\overline{9}$-brane pairs $N=2^{k-1}$, and which also leads to a particularly natural representative of the tachyon vortex configuration. The tachyon vortex that (at least locally in $X$ ) represents the D-brane wrapping a manifold $Y$ of codimension $2 k$ in $X$ is constructed as follows. The group of rotations $S O(2 k)$ in the dimensions normal to $Y$ in $X$ has two inequivalent spinor representations, which give rise to two spinor bundles $\mathcal{S}_{+}$and $\mathcal{S}_{-}$ on $Y$. These bundles can be extended to a neighborhood of $Y$ in $X$, and therefore they define (modulo possible global obstructions that can typically be eliminated by pair creating extra 9 -branes and $\overline{9}$-branes) a K-theory class $\left[\mathcal{S}_{+}\right]-\left[\mathcal{S}_{-}\right]$. In the construction of the bound state, we identify $\mathcal{S}_{+}$with the Chan-Paton bundle carried by 9 -branes, and $\mathcal{S}_{-}$with the bundle supported by $\overline{9}$-branes. The gauge symmetry on the 9 -brane worldvolume is $U\left(2^{k-1}\right) \times$ $U\left(2^{k-1}\right)$. The tachyon is a map from $\mathcal{S}_{+}$to $\mathcal{S}_{-}$, and its vortex configuration of vorticity one around $Y$ can be written in a very simple form using $\Gamma$ matrices of $S O(2 k)$ (which represent maps from $\mathcal{S}_{+} \oplus \mathcal{S}_{-}$to itself), as

$$
T=\Gamma_{m} x^{m}
$$

where $x^{m}, m=1, \ldots 2 k$ are coordinates in the directions transverse to $Y$ in a small neigborhood of $Y$ in spacetime. We have omitted a multiplicative convergence factor in 2.3 , which approaches one in the small vicinity of $Y$ and goes to zero as we approach infinity, ensuring that far away from the core of the vortex, $T$ takes values in the vacuum manifold 2.2 , with $N=2^{k-1}{ }^{2}$

As an example, let us consider the case of codimension two. The gauge symmetry is $U(1) \times U(1)$, and the tachyon vortex of vorticity one can be written as

$$
T=\sigma_{i} x^{i}=\left(\begin{array}{cc}
0 & x^{1}+i x^{2} \\
x^{1}-i x^{2} & 0
\end{array}\right)
$$

\footnotetext{
${ }^{2}$ Our discussion has been local in $X$; when global topology is taken into account, one sometimes has to "stabilize" (in the K-theory sense) the configuration of 9-branes and $\overline{9}$-branes by pair creating extra 9 -branes and $\overline{9}$-branes, thus leading to a configuration of 9-brane $\overline{9}$-brane pairs described by $\left(\mathcal{S}_{+} \oplus H, \mathcal{S}_{-} \oplus H\right)$; for more details, see [2] .
} 
where we have used a particular convenient representation of the two $\Gamma$ matrices $\sigma_{1,2}$ in two transverse dimensions $x^{1,2}$.

This bound-state construction defines a map $\mathrm{K}(Y) \rightarrow \mathrm{K}(X)$ for any submanifold $Y$ of $X$ that admits a $\operatorname{Spin}_{c}$ structure [2] . Some other details of this construction, together with more details about its relation to the Thom isomorphism, the Gysin map, and the Atiyah-Bott-Shapiro construction in $\mathrm{K}$-theory, can be found in [2] . For some general K-theory background, see [22]-[26].

\subsection{Type IIA on $X$ and $\mathrm{K}^{-1}(X)$}

It has been suggested in [2] that D-brane charges in Type IIA theory should be similarly classified by a certain higher $\mathrm{K}$-theory group $\mathrm{K}^{-1}(X)$. This conjecture is supported by the following argument. Consider the reduced $\mathrm{K}$-theory groups of spheres, $\widetilde{\mathrm{K}}\left(\mathbf{S}^{n}\right)$. These groups classify possible $(9-n)$ branes in Type IIB theory on $\mathbf{R}^{10}$ [2] . Using Bott periodicity, one can show that $\widetilde{\mathrm{K}}\left(\mathbf{S}^{2 n}\right)=\mathbf{Z}$ and $\widetilde{\mathrm{K}}\left(\mathbf{S}^{2 n+1}\right)=0$. The higher K-theory group $\mathrm{K}^{-1}(X)$ will be defined precisely below, but now we only invoke the fact that

$$
\mathrm{K}^{-1}\left(\mathbf{S}^{n}\right)=\tilde{\mathrm{K}}\left(\mathbf{S}^{n+1}\right) .
$$

Hence, $\mathrm{K}^{-1}\left(\mathbf{S}^{2 n+1}\right)=\mathbf{Z}$ and $\mathrm{K}^{-1}\left(\mathbf{S}^{2 n}\right)=0$. This is in accord with the fact that Type IIB theory contains supersymmetric $p$-branes for $p$ odd, while Type IIA theory has $p$-branes with $p$ even.

Thus, the higher $\mathrm{K}$-theory group $\mathrm{K}^{-1}(X)$ of spacetime $X$ is a natural candidate for the $\mathrm{K}$-theory group that classifies D-brane charges in Type IIA theory. For a manifold $X$ of dimension $d$, the higher $\mathrm{K}$-theory group $\mathrm{K}^{-1}(X)$ is usually defined using the ordinary K-theory group of a $d+1$ dimensional extension $X^{\prime}$ of $X$. If $X$ is a spacetime manifold of string theory, $X^{\prime}$ will be eleven-dimensional, and we may suspect a connection to M-theory. The definition of $\mathrm{K}^{-1}(X)$ that is most suggestive of M-theory sets $X^{\prime}=X \times \mathbf{S}^{1}$, and defines $\mathrm{K}^{-1}(X)$ using the $\mathrm{K}$-theory group $\tilde{\mathrm{K}}\left(X \times \mathbf{S}^{1}\right)$. More precisely, $\mathrm{K}^{-1}(X)$ is defined as the subgroup in $\widetilde{\mathrm{K}}\left(X \times \mathbf{S}^{1}\right)$ that maps to the trivial class in $\widetilde{\mathrm{K}}(X)$, by the map induced from the embedding of $X$ as $X \times$ point in $X \times \mathbf{S}^{1}{ }^{3}$ This definition of $\mathrm{K}^{-1}(X)$ that uses $X^{\prime}=X \times \mathbf{S}^{1}$ is somewhat awkward, and we can define $\mathrm{K}^{-1}(X)$ more directly by choosing a slightly

\footnotetext{
${ }^{3}$ The purpose of this extra condition is to eliminate elements in $\widetilde{\mathrm{K}}\left(X \times \mathbf{S}^{1}\right)$ that correspond to $\widetilde{\mathrm{K}}(X)$. In the string theory language, since $\mathrm{K}^{-1}\left(X \times \mathbf{S}^{1}\right) \cong \mathrm{K}^{-1}(X) \oplus \widetilde{\mathrm{K}}(X)$, the restriction of $\widetilde{\mathrm{K}}\left(X \times \mathbf{S}^{1}\right)$ to its subgroup $\mathrm{K}^{-1}(X)$ eliminates charges that would correspond in Type IIA string theory to stable $p$-branes with $p$ odd.
} 
different $X^{\prime}$ as follows. Consider first the product of $X$ with a unit interval, $X \times I$, and define the so-called "suspension" $S^{\prime}(X)$ of $X$ by identifying all points in each boundary component of $X \times I$. Thus, for example, the suspension of the $m$-sphere $\mathbf{S}^{m}$ is the $(m+1)$-dimensional sphere, $S^{\prime}\left(\mathbf{S}^{m}\right)=$ $\mathrm{S}^{m+1}$. One can define $\mathrm{K}^{-1}(X)$ by starting with $X^{\prime}=S^{\prime}(X)$, and setting

$$
\mathrm{K}^{-1}(X)=\tilde{\mathrm{K}}\left(S^{\prime}(X)\right) .
$$

In the particular case of $X=\mathbf{S}^{m}$, we obtain 2.5 .

In Type IIB theory, the fact that $\tilde{\mathrm{K}}(X)$ classifies D-brane charges leads to the construction of all possible D-branes as bound states of spacetimefilling 9-brane $\overline{9}$-brane pairs, reviewed briefly in the previous section. When we try to extend this construction of $\mathrm{K}(X)$ from Type IIB theory to an analogous construction of the $\mathrm{K}^{-1}(X)$ groups on the Type IIA side, we encounter the following difficulty. As we have seen, $\mathrm{K}^{-1}(X)$ is defined as the usual K-theory group of an extended manifold $S^{\prime}(X)$. In string theory, $X$ is a spacetime manifold of dimension ten, and therefore its extension $S^{\prime}(X)$ used in the definition of $\mathrm{K}^{-1}(X)$ is eleven-dimensional. This indeed suggests a possible relation to M-theory (as pointed out in [2]), but since we do not have a hierarchy of D-branes in M-theory (and in particular there are no 10-branes), it is unclear how to interpret the $\mathrm{K}^{-1}(X)$ group that should classify D-brane charges of Type IIA string theory but is defined using an eleven-dimensional extension of $X$.

In the next section we will present a string theory construction (as opposed to an M-theory construction) of all supersymmetric D-branes of Type IIA string theory, similar in spirit to that of $[1,2]$. The unstable 9-branes of Type IIA theory, introduced in section 1, will provide the crucial ingredient for our construction. We will see that this stringy construction is a translation of one possible definition of $\mathrm{K}^{-1}(X)$ in $\mathrm{K}$-theory. Hence, our construction proves that the D-brane charges of Type IIA string theory are indeed classified by $\mathrm{K}^{-1}(X)$, as suggested by Witten in [2] . The argument can be easily generalized to see that $D$-branes in Type $\mathrm{I}^{\prime}$ theory are similarly classified by $\mathrm{KR}^{-1}(X)$. 


\section{Type IIA D-Branes as Bound States of Unstable 9-Branes}

\subsection{Unstable 9-Branes in Type IIA Theory}

We have pointed out in section 1 that once we relax the condition that D-branes be supersymmetric and carry a $\mathrm{RR}$ charge, we can construct $\mathrm{D} p$ branes of any $p \leq 9$, at the cost of sometimes obtaining unstable configurations. In particular, in Type IIA theory we can construct a spacetime-filling 9-brane. Its structure can be easily understood from the form of its boundary state $|B\rangle$, which - as a particular coherent state in the Hilbert space of the Type IIA closed string - represents the boundary conditions on the closed string annihilated into the 9-brane. The D9-brane boundary state (we will only consider the 9-brane of Type IIA theory, generalizations to $(2 k-1)$-branes of lower dimensions are obvious) is thus given by

$$
|B\rangle=|B,+\rangle_{\mathrm{NS} \mathrm{NS}}-|B,-\rangle_{\mathrm{NS} \mathrm{NS}} .
$$

Here $|B, \pm\rangle_{\text {NS NS }}$ represents the two possible implementations of the Neumann boundary conditions on all spacetime coordinates, [31]-[33].

There is no RR component in the boundary state, as none is invariant under the Type IIA GSO projection in the closed string channel. Indeed, there are two RR states $|B, \pm\rangle_{\mathrm{RR}}$ that implement Neumann boundary conditions on all coordinates. These states transform into each other under the worldsheet fermion number operators $(-1)^{F_{L, R}}$, as follows (see e.g. [8] ):

$$
(-1)^{F_{L}}|B, \pm\rangle_{\mathrm{RR}}=|B, \mp\rangle_{\mathrm{RR}}, \quad(-1)^{F_{R}}|B, \pm\rangle_{\mathrm{RR}}=|B, \mp\rangle_{\mathrm{RR}}
$$

However, the GSO projection in Type IIA theory chooses opposite chiralities in the left-moving and the right-moving sector, and no combination of $|B, \pm\rangle_{\mathrm{RR}}$ is invariant under the Type IIA GSO operator $\left(1-(-1)^{F_{L}}\right)(1+$ $\left.(-1)^{F_{R}}\right){ }^{4}$ The absence of a RR boundary state means that no RR tadpole is associated with our 9-brane, and therefore, no spacetime anomalies related to RR tadpoles can arise. Unlike in Type IIB theory, where tadpole cancellation requires an equal number of 9 -branes and $\overline{9}$-branes, we do not get a restriction on the number of 9-branes from tadpole cancellation. These 9 -branes carry no conserved charge, and there is no distinction between a 9-brane and an anti-9-brane.

Since there is no RR component in the boundary state, there is no GSO projection in the dual, open-string channel of the toroidal amplitude $\langle B \mid B\rangle$.

\footnotetext{
${ }^{4} \mathrm{I}$ am grateful to Oren Bergman for discussions on this subject.
} 
Therefore, the open string connecting one such 9-brane to itself will contain - in the NS sector - both the $U(1)$ gauge field that a supersymmetric brane would carry, and the tachyon field $T$ that would, in the case of supersymmetric branes, be projected out by the GSO projection. In the Ramond sector of the open string, both spacetime chiralities of the ground state spinor are retained, again due to the absence of any GSO projection.

A more precise way of implementing this boundary-state construction of unstable D-branes in a way compatible with the general Type IIA GSO projections on higher-genus worldsheets has been proposed in a similar case of unstable Type IIB D0-branes by Witten in [2] . In this procedure, one introduces an extra fermion $\eta$ at each boundary component that corresponds to the string worldsheet ending on the 9-brane. (Similar boundary fermions were introduced some time ago in a different context in [28] ). This extra fermion is described by the Lagrangian $\int \eta(d \eta / d t) d t$, with $t$ a periodic coordinate along the worldsheet boundary component. Quantization of this fermion gives an extra factor of $\sqrt{2}$ in the sector with antiperiodic boundary conditions, and the zero mode of $\eta$ kills the contribution to the worldsheet path integral from the $\mathrm{R}$ sector with periodic boundary conditions. In terms of boundary states, this indeed reproduces our boundary state $|B\rangle$, but now not because there would be no GSO projection. Instead, there is a GSO projection, the Ramond boundary state $|B, R\rangle$ vanishes identically, and the total boundary state can be rewritten as

$$
|B\rangle=\frac{1}{\sqrt{2}}(\sqrt{2}|B\rangle+|B, R\rangle) .
$$

The extra factor of $\sqrt{2}$ comes from the extra Chan-Paton factor associated with the extra boundary fermion $\eta$.

In the presence of Type IIA $2 p$-branes carrying RR charges, there would be a sector of open strings connecting the $2 p$-brane to the 9-brane. As in the case of Type IIB D0-branes studied in [2], the worldsheet rules for calculating amplitudes in such cases also require the presence of the extra boundary fermion $\eta$.

More generally, consider $N$ coincident 9-branes. The free open-string spectrum of massless and tachyonic states gives rise to the following lowenergy field content on the spacetime-filling worldvolume,

$$
A_{\mu}, T, \chi \text {, and } \chi^{\prime},
$$

where $A_{\mu}$ is a $U(N)$ gauge field, $T$ is a tachyon field in the adjoint of $U(N)$, and the two chiral fermions $\chi, \chi^{\prime}-$ also in the adjoint of $U(N)$ - carry oppo- 
site spacetime chiralities. ${ }^{5}$ This should be contrasted with the worldvolume field content of the Type IIB system of $N$ pairs of 9-branes and $\overline{9}$-branes, where the bosonic sector 2.1 consists of a $U(N) \times U(N)$ gauge field and a tachyon in $(N, \bar{N})$.

Notice the intriguing fact that this field content 3.4 on $N$ 9-branes of Type IIA theory coincides with the ten-dimensional decomposition of a system in eleven dimensions, consisting of a $U(N)$ gauge field $\mathcal{A}_{M}$ and a 32-component spinor $\Psi$ in the adjoint of $U(N)$. In particular, the adjoint tachyon plays the role of an eleventh component of the $U(N)$ gauge field, and the ten-dimensional decomposition gives 3.4 as

$$
\mathcal{A}_{M}=\left(A_{\mu}, T\right), \quad \Psi=\left(\chi, \chi^{\prime}\right) .
$$

Of course, this hidden eleven-dimensional symmetry of the lowest open-string states is broken already at the level of free fields by the tachyon mass.

\subsection{9-Brane Configurations and $\mathrm{K}^{-1}(X)$}

In analogy with our understanding of Type IIB D-branes in K-theory, we want to achieve two separate things: (1) classification of branes in Type IIA theory on general $X,(2)$ construction of branes in terms of bound states of higher-dimensional branes.

First, we will consider possible configurations of $N$ 9-branes in Type IIA string theory, up to possible creation and annihilation of 9-branes from and to the vacuum.

Recalling our discussion in Section 1 of a system of such unstable 9branes in Type IIA theory, we expect that the system will rapidly decay to the supersymmetric vacuum, whenever it does not carry lower-dimensional D-brane charges. We will call such 9-brane configurations "elementary." Any such "elementary" configuration of $N^{\prime}$ branes wrapping $X$ will give rise to a $U\left(N^{\prime}\right)$ bundle $F$, together with a $U\left(N^{\prime}\right)$ gauge field on $F$ and a tachyon $T$ in the adjoint representation of $U\left(N^{\prime}\right)$. The bound-state construction that we discuss below indicates that the presence or absence of lower D-brane charges can be measured by the tachyon condensate $T$.

Thus, we will assume (cf. $[1,2])$ that a bundle $E$ with tachyon field $T$ can be deformed - by processes that involve only creation and annihilation

\footnotetext{
${ }^{5}$ In the simplest case of just one 9-brane, the gauge group is $U(1)$, and the tachyon is a real scalar field. We will see below that this case is somewhat degenerate, and one may want to "stabilize" it - in the sense of K-theory - by embedding this system into a larger system with more than one 9-brane.
} 
of "elementary" 9-branes - into a bundle isomorphic to $E \oplus F$, with $F$ the Chan-Paton bundle of an elementary 9-brane configuration.

This definition of equivalence classes of 9-branes with tachyon condensate, up to creation or annihilation of "elementary" 9-brane configurations from and to the vacuum, corresponds to the following construction in $\mathrm{K}$ theory.

It turns out [22] that in K-theory, one can define the higher K-theory group $\mathrm{K}^{-1}(X)$ without using an eleven-dimensional extension of $X$. Instead, one starts with pairs $(E, \alpha)$, where $E$ is a $U(N)$ bundle for some $N$, and $\alpha$ is an automorphism on $E$. (In fact, we do not lose generality if we consider only trivial bundles $E$ on $X$.) A pair $(F, \beta)$ is called "elementary" if the automorphism $\beta$ can be continuously deformed to the identity automorphism on $F$, within automorphisms of $F$. One defines an equivalence relation on pairs $(E, \alpha)$, as follows. Two pairs $\left(E_{1}, \alpha_{1}\right)$ and $\left(E_{2}, \alpha_{2}\right)$ are equivalent if there are two elementary pairs $\left(F_{1}, \beta_{1}\right)$ and $\left(F_{2}, \beta_{2}\right)$ such that

$$
\left(E_{1} \oplus F_{1}, \alpha_{1} \oplus \beta_{1}\right) \cong\left(E_{2} \oplus F_{2}, \alpha_{2} \oplus \beta_{2}\right) .
$$

The set of all such equivalence classes of pairs $(E, \alpha)$ on $X$ is a group: the inverse element to the class of $(E, \alpha)$ is the class of $\left(E, \alpha^{-1}\right)$. This group of classes of pairs $(E, \alpha)$ on $X$ is precisely $\mathrm{K}^{-1}(X)$ (as defined e.g. in [22] , Section II.3). This "string theory" definition of $\mathrm{K}^{-1}(X)$ - which uses bundles with automorphisms on the ten-dimensional spacetime $X$ - is equivalent to the definition of $\mathrm{K}^{-1}(X)$ reminiscent of M-theory (and reviewed in section 2.2) which uses pairs of bundles on the eleven-dimensional extension $X \times \mathbf{S}^{1}$. This rather non-trivial fact can be found e.g. in [22] , Theorem II.4.8.

In string theory, the role of the $N$-dimensional bundle $E$ is played by the Chan-Paton bundle carried by a system of $N$ unstable Type IIA 9branes. The automorphism $\alpha$ is a little harder to see directly in the 9brane. However, we will see below that in the bound-state construction of supersymmetric D-branes as bound states in a system of 9-branes, the role of $\alpha$ is played by

$$
\mathcal{U}=-e^{\pi i T},
$$

where $T$ is the adjoint $U(N)$ tachyon on the 9-brane worldvolume. Elementary pairs $(F, \alpha)$ correspond to elementary brane configurations that do not carry any lower-dimensional D-brane charge, and therefore can be created from and annihilated to the vacuum. Thus, possible 9-brane configurations up to creation and annihilation of "elementary" 9-branes are classified by $\mathrm{K}^{-1}(X)$. This, together with our explicit bound-state construction below, demonstrates that $\mathrm{K}^{-1}(X)$ indeed classifies D-brane charges in Type IIA theory. 
In contrast to Type IIB theory, where one is supposed to consider tadpole cancelling configuration of an equal number of 9-branes and $\overline{9}$-branes, in Type IIA theory there is no such restriction on the number of 9-branes. This statement has a nice interpretation in K-theory. In Type IIB theory, tadpole cancelling configurations of 9 -brane $\overline{9}$-brane pairs correspond to the reduced $\mathrm{K}$-theory group, $\widetilde{K}(X)$, related to the full $\mathrm{K}(X)$ by $\mathrm{K}(X)=\mathbf{Z} \oplus \widetilde{\mathrm{K}}(X)$. One can define a "reduced" higher K-theory group $\widetilde{\mathrm{K}}^{-1}(X)[22,26]$, but it turns out that (for the class of spacetime manifolds that one encounters in string theory) $\widetilde{\mathrm{K}}^{-1}(X)$ is always equal to $\mathrm{K}^{-1}(X)$.

\subsection{Type IIA D-Branes as Bound States of 9-Branes}

So far, we have suggested a classification of all configurations of 9-branes up to creation or annihilation of "elementary" 9-branes that do not carry any lower D-brane charge. Here we present a construction that allows one to embed any lower-dimensional branes into a system of 9-branes in Type IIA theory: thus, just as in Type IIB theory [2], whatever can be done with stable lower-dimensional branes can be done with unstable 9-branes of Type IIA theory.

Even though we will mostly focus on bound states of unstable 9-branes, one could also start with any lower-dimensional unstable $(2 k-1)$-branes, and construct stable $2 p$-branes for $p \leq k-1$ as their bound states. In turn, each such lower-dimensional unstable $(2 k-1)$-brane can be viewed as an unstable bound state of a $2 k$-brane $\overline{2 k}$-brane pair, and we obtain a whole hierarchy of bound-state constructions, corresponding to a hierarchy of $\mathrm{K}$-theory isomorphisms. However, the only truly interesting case is that of 9-branes, for the following reason. Using the techniques of [2] , each individual lowerdimensional stable $\mathrm{D} p$-brane of Type IIA theory can already be constructed as a bound state of a certain number of 8-brane $\overline{8}$-brane pairs. There seems to be no gain in representing this $\mathrm{D} p$-brane for example as a bound state of unstable 7-branes. However, while not every configuration of stable Dbranes of Type IIA fits into the worldvolume of a given 8-brane $\overline{8}$-brane system, it clearly fits into the worldvolume of a system of spacetime-filling 9-branes. This embedding will enable us to keep all spacetime symmetries manifest, and will not lead to any kinematical restrictions on configurations of lower-dimensional stable $\mathrm{D} p$-branes that can be studied this way.

Consider first a single unstable 9-brane in Type IIA theory. The gauge group is $U(1)$, and the tachyon is just a real scalar field of charge zero. We do not know the exact form of the tachyon potential $V(T)$, but we can assume that $V(T)=V(-T)$, and that $T$ will condense into one of two vacuum 
values, $T= \pm T_{0}$.

We will assume - in close analogy with a similar assumption made in [1] in the related case of pairs of stable $p$-branes and $\bar{p}$-branes - that when the tachyon condenses into either $T_{0}$ or $-T_{0}$, the negative energy density associated with the condensate will cancel the positive energy density associated with the 9-brane tension, and the 9-brane will completely annihilate into the supersymmetric vacuum. This 9-brane is an example of what we called an "elementary" 9-brane in the previous section.

Since the vacuum manifold of the tachyon field consists of two points $\pm T_{0}$, the tachyon can form a kink of codimension one in spacetime. Near the core of the kink, the tachyon field will be (up to a convergence factor, assuring that $T \rightarrow \pm T_{0}$ asymptotically at infinity)

$$
T= \pm x^{9},
$$

where $x^{9}$ is the coordinate normal to the core of the kink. Thus, the core of this kink represents a domain wall in spacetime, which we will interpret as the supersymmetric 8 -brane or $\overline{8}$-brane, depending on the sign in 3.8 (or, in other words, the sign of the difference between the asymptotic vacuum values of the tachyon $T(-\infty)-T(+\infty)$ on the two sides of the domain wall). Notice that only one 8 -brane or one $\overline{8}$-brane can be constructed from one 9-brane.

Consider now $N$ unstable 9-branes. The gauge symmetry is $U(N)$, and the tachyon is in the adjoint of the gauge group. The tachyon will again have the tendency to roll down to a certain value $T_{0}$ at a minimum of its potential, possibly breaking a part of the $U(N)$ gauge symmetry. The precise pattern of symmetry breaking depends on the structure of eigenvalues of $T_{0}$, which in turn depends on the precise form of the tachyon potential, which of course is not known. It is natural to expect that $V(T)=V(-T)$, and that all eigenvalues of $T_{0}$ are equal to a certain $T_{v}$, possibly up to a sign. It is easy to see that this structure of eigenvalues would be obtained for example from even potentials of the form

$$
V(T)=-m^{2} \operatorname{tr}\left(T^{2}\right)+\lambda^{2} \operatorname{tr}\left(T^{4}\right)+\ldots,
$$

and with each term containing only a single trace. Such terms in the potential are expected from the disc amplitudes, i.e. at tree level in open-string perturbation theory.

When the tachyon on the worldvolume of $N$ 9-branes condenses into the vacuum value with $N-k$ positive eigenvalues and $k$ negative ones,

$$
T_{0}=T_{v}\left(\begin{array}{cc}
1_{N-k} & 0 \\
0 & -1_{k}
\end{array}\right),
$$


the $U(N)$ gauge symmetry is broken to $U(N-k) \times U(k)$. Just as in the case of a single 9-brane, the tachyon field can form kinks of codimension one. One particularly interesting case corresponds to the kink in all eigenvalues of $T$, localized at a common domain wall $Y$ of codimension one in spacetime, which near $Y$ can be written (again, up to a convergence factor), as

$$
T=\left(\begin{array}{cc}
x^{9} \cdot 1_{N-k} & 0 \\
0 & -x^{9} \cdot 1_{k}
\end{array}\right)
$$

We conjecture that this configuration should be interpreted as $N-k$ 8-branes and $k \overline{8}$-branes with coinciding wordvolumes wrapping $Y{ }^{6}$ More general configurations of separated 8-branes and $\overline{8}$-branes can be constructed by letting each eigenvalue vanish along a separate manifold of codimension one in spacetime.

Thus, any number of 8-branes and $\overline{8}$-branes can be constructed from 9branes, but one cannot represent more than $N$ of them as a bound state of $N$ 9-branes: if we want to add an extra 8-brane, the construction has to be "stabilized" in the sense of K-theory, by adding an extra 9-brane.

In general, worldsheets with more than one boundaries could give rise to corrections to the tachyon potential 3.9 , of the form

$$
\tilde{\lambda}^{2}\left(\operatorname{tr}\left(T^{2}\right)\right)^{2}+\ldots
$$

with more than one trace in each individual term. Using the analysis of section III.D. and Appendix B of [29] , one can show that even in the case of a generic potential 3.9 and 3.12 with $\lambda^{2} \geq 0, \widetilde{\lambda}^{2}>0$, the minimum $T_{0}$ of the tachyon potential still has only two eigenvalues, $\pm T_{0}$, and the vacua with different values of $k$ stay degenerate. ${ }^{7}$

One could now combine the construction 3.11 of 8-branes and $\overline{8}$-branes from 9-branes with the construction discussed in [2, 1], and construct all lower-dimensional D-2p-branes as bound states of a sufficient number of 8 brane $\overline{8}$-brane pairs prepared from 9 -branes.

This two-step procedure has several significant drawbacks. First of all, we have to select a preferred submanifold of codimension one in spacetime,

\footnotetext{
${ }^{6}$ The interpretation of this multiple kink configuration as a set of 8 -branes and $\overline{8}$-branes suggests the existence of a coupling

$$
\int_{X} C_{9} \wedge \operatorname{tr}(d T)
$$

between the spacetime RR 9-form $C_{9}$ and the $U(N)$ tachyon $T$ on the 9 -brane worldvolume $X$.

${ }^{7}$ I wish to thank John Preskill for bringing Ref. [29] to my attention.
} 
which represents the worldvolume of the 8 -brane $\overline{8}$-brane system. This breaks some of the manifest spacetime symmetries. Perhaps more importantly, configurations of lower-dimensional branes that cannot be embedded into the worldvolume of a single system of coincident 8-brane $\overline{8}$-brane pairs may require - when realized via the two-step construction involving 8-branes - that extra 9-branes be introduced, due to the fact that each 8-brane or $\overline{8}$-brane needs its own 9-brane. This would make the number of 9-branes used in the bound-state construction artificially dependent on the number and precise configuration of the lower-dimensional bound state.

These shortcomings will be resolved in a one-step procedure, in which we construct arbitrary lower-dimensional D-2p-brane directly as a bound state of a system of 9-branes. This one-step procedure avoids the intermediate step involving 8-branes and $\overline{8}$-branes, and therefore avoids the degeneracy of the codimension-one bound-state construction, leading to a more powerful description of lower-dimensional D-branes as bound states. Along the way, we will discover many intriguing connections to K-theory.

\section{The General Bound State Construction}

From now on, we will consider 9-brane systems whose tachyon condensate $T_{0}$ has an equal number of positive and negative eigenvalues. Thus, the number of 9-branes is $2 N$ for some $N$, and the gauge group $U(2 N)$ is broken to $U(N) \times U(N)$. The vacuum manifold is

$$
\mathcal{V}_{1}(2 N)=U(2 N) /(U(N) \times U(N))
$$

We are interested in stable, vortex-like configurations in the tachyon field. Away from the core of such a stable vortex, the tachyon field (almost) assumes its vacuum values. This defines a map of the sphere $\mathbf{S}^{m}$ surrounding the core of a vortex of codimension $m+1$ into the vacuum manifold $\mathcal{V}_{1}(2 N)$. Possible candidates for stable tachyon vortices in this codimension are thus classified by elements of the homotopy group $\pi_{m}\left(\mathcal{V}_{1}(2 N)\right)$. It turns out that homotopy groups of the vacuum manifold 3.13 are non-trivial in even dimensions, $\pi_{2 k}\left(\mathcal{V}_{1}(2 N)\right)=\mathbf{Z}$, and trivial in all odd dimensions. (Here we are assuming that $N$ is large enough, so that it belongs to the "stable" range.) This should be contrasted with the case of Type IIB 9-brane $\overline{9}$-brane pairs, reviewed in section 2.1: in the case of Type IIB 9-branes, the vacuum manifold $\mathcal{V}_{0}(N)=U(N)$ has non-zero homotopy groups only in odd dimensions, $\pi_{2 k-1}\left(\mathcal{V}_{0}(N)\right)=\mathbf{Z}$. Therefore, while the Type IIB system of 9-brane $\overline{9}$-brane pairs supports bound states of codimension $2 k$, our Type IIA 9-brane system will exhibit bound states in codimensions $2 k+1$. 
This structure of homotopy groups is not coincidental, and in fact reflects a deep connection of our construction to K-theory. Our vacuum manifold $\mathcal{V}_{1}(2 N)$ can be thought of as a Grassmannian manifold whose points are $N$-dimensional complex subspaces in $\mathbf{C}^{2 N}$. This Grassmannian plays an important role in $\mathrm{K}$-theory, as it represents a standard finite-dimensional approximation to the "universal classifying space" $B U$ (see e.g. [23]). The importance of $B U$ in K-theory stems from the fact that the K-theory group $\widetilde{\mathrm{K}}(X)$ is canonically isomorphic, for any (reasonable) $X$, to the set of homotopy classes of maps from $X$ to this universal classifying space,

$$
\widetilde{\mathrm{K}}(X)=[X, B U] .
$$

Similarly, the higher $\mathrm{K}$-theory group $\mathrm{K}^{-1}(X)$ is related to the set of homotopy classes of maps from $X$ to the infinite unitary group $U$,

$$
\mathrm{K}^{-1}(X)=[X, U] \text {. }
$$

Thus, the vacuum manifold $\mathcal{V}_{1}(N)$ of the tachyon on Type IIA 9-branes is a finite-dimensional approximation to the classifying space $B U$, and the vacuum manifold $\mathcal{V}_{0}(N)=U(N)$ of the tachyon in the Type IIB 9-brane $\overline{9}$-brane system is a finite-dimensional approximation to the infinite unitary group $U$.

The structure of homotopy groups of the tachyon vacuum manifold $\mathcal{V}_{1}(2 N)$ indicates the possibility of bound states in all odd codimensions on the worldvolume of Type IIA 9-branes on $\mathbf{R}^{10}$. These bound states will appear as tachyon vortices, and will be interpreted as supersymmetric $\mathrm{D}$ $2 p$-branes of Type IIA theory. Just like in the case of Type IIB theory [2] , K-theory suggests the number of Type IIA 9-branes that is particularly natural for the bound state construction. Bound states of codimension $2 k+1$ are most efficiently described by $2 N=2^{k} 9$-branes. Stable tachyon vortices in this codimension are classified by the $2 k$-th homotopy group of the vacuum manifold, $\pi_{2 k}\left(\mathcal{V}_{1}\left(2^{k}\right)\right)=\mathbf{Z}$. In fact, homotopy groups of $\mathcal{V}_{1}$ are related to the homotopy groups of $U(N)$, via ${ }^{8}$

$$
\begin{aligned}
\ldots=\pi_{2 k+1}\left(U\left(2^{k}\right)\right) & =\pi_{2 k}\left(U\left(2^{k}\right) / U\left(2^{k-1}\right) \times U\left(2^{k-1}\right)\right) \\
& =\pi_{2 k-1}\left(U\left(2^{k-1}\right)\right)=\ldots
\end{aligned}
$$

The tachyon vortex corresponding to the generator of $\pi_{2 k}\left(\mathcal{V}_{1}\right)$ can be explicitly constructed as follows. The worldvolume of $2^{k} 9$-branes supports a

\footnotetext{
${ }^{8}$ This is precisely one half of the statement of Bott periodicity $[22,26,30]$. The other half of Bott periodicity similarly relates odd homotopy groups of $\mathcal{V}_{1}$ and even homotopy groups of $\mathcal{V}_{0}$. Using these relations, together with 3.14 and 3.15 , one can for example derive all $\mathrm{K}$-theory groups of spheres, used in section 2.2 to classify supersymmetric Type II D-branes in $\mathbf{R}^{10}$.
} 
$U\left(2^{k}\right)$ Chan-Paton bundle, which we identify with the spinor bundle $\mathcal{S}$ of the group $S O(2 k+1)$ of rotations in the transverse dimensions. The tachyon condensate is then given by the vortex configuration

$$
T(x)=\Gamma_{m} x^{m}
$$

As in the Type IIB case [2],$\Gamma_{m}$ are the $\Gamma$-matrices of the group of rotations in transverse dimensions $x^{m}, m=1, \ldots, 2 k+1.3 .17$ describes a stable vortex in codimension $2 k+1$, which we interpret as the supersymmetric $(8-2 k)$-brane of Type IIA theory.

Even though the expression for the tachyon vortex 3.17 on Type IIA 9 -branes looks formally identical to the tachyon vortex 2.3 on the system of 9-brane $\overline{9}$-brane pairs of Type IIB theory, there is a significant difference between 3.17 and 2.3 . Asymptotically away from the vortex of the tachyon, 3.17 takes values in the vacuum manifold $U\left(2^{k}\right) /\left(U\left(2^{k-1}\right) \times U\left(2^{k-1}\right)\right)$. On the other hand, the Type IIB vortex 2.3 takes asymptotically values in $\left(U\left(2^{k-1}\right) \times U\left(2^{k-1}\right)\right) / U\left(2^{k-1}\right)$. One can see this distinction clearly in $\mathrm{K}-$ theory, where the two tachyon condensates represent generators of disctinct $\mathrm{K}$-theory groups; 2.3 generates the relative group $\mathrm{K}\left(\mathbf{B}^{2 k}, \mathbf{S}^{2 k-1}\right.$ ) (with $\mathbf{B}^{2 k}$ a ball $|x|^{2} \leq 1$ in $\left.\mathbf{R}^{2 k}\right)$, and 3.17 represents the generator of $\mathrm{K}^{-1}\left(\mathbf{B}^{2 k+1}, \mathbf{S}^{2 k}\right)$ $[22,24,25]$.

The tachyon vortex 3.17 is accompanied by a non-trivial $U\left(2^{k}\right)$ gauge field, due to the finite energy condition imposed on the whole configuration. The non-triviality of the unbroken part of the gauge field is measured by the element in $\pi_{2 k-1}\left(U\left(2^{k-1}\right)\right)$ that maps to the element of $\pi_{2 k}\left(\mathcal{V}_{1}\left(2^{k}\right)\right)$ corresponding to the tachyon condensate 3.17 , under the isomorphism of homotopy groups 3.16 . In the construction of the gauge field, one starts with topologically trivial gauge fields on the upper and lower hemisphere of $\mathbf{S}^{2 k}$, and the element of $\pi_{2 k-1}\left(U\left(2^{k-1}\right)\right)$ corresponds to a large gauge transformation along the equator of $\mathbf{S}^{2 k}$ that allows one to patch the gauge fields on the two coordinate systems into a gauge field carrying the corresponding topological charge on $\mathbf{S}^{2 k}$. This is a standard construction known from the physics of magnetic monopoles [34], and the unbroken long-range gauge field of $U(N) \times U(N)$ indeed corresponds to that of a generalized magnetic monopole. The corresponding magnetic charge is measured by the $k$-th Chern class of the gauge bundle on $\mathbf{S}^{2 k}$ (Some relevant background on magnetic monopoles can be found in $[35,36]$.)

The other side of the isomorphism 3.16 , which relates $\pi_{2 k}\left(\mathcal{V}_{1}\left(2^{k}\right)\right)$ to $\pi_{2 k+1}\left(U\left(2^{k}\right)\right)$, is also important: it allows us to relate our bound-state construction to the definition of $\mathrm{K}^{-1}(X)$. Given the tachyon condensate 
$T=\Gamma \cdot x$, we can construct an element of $\pi_{2 k+1}\left(U\left(2^{k}\right)\right)$ as follows. Consider

$$
\mathcal{U}=-e^{\pi i T} .
$$

Since the tachyon is in the adjoint of $U\left(2^{k}\right), \mathcal{U}$ defines a map from the unit ball $|x| \leq 1$ to $U\left(2^{k}\right)$. The group $U\left(2^{k}\right)$ as well as its Lie algebra can be represented by $2^{k} \times 2^{k}$ matrices which are unitary and hermitian, respectively. We will use a particularly useful description of the coset 3.13 , as the set of all $2^{k} \times 2^{k}$ matrices that are simultaneously hermitian and unitary [30] . In particular, all such matrices square to one, as elements in $U\left(2^{k}\right)$. (Incidentally, this proves that far from the core of the vortex, for an appropriate convergence factor $f(|x|)$ omitted in 3.17 , the tachyon condensate 3.17 indeed takes values in the vacuum manifold $\mathcal{V}_{1}\left(2^{k}\right)$.)

We can now apply this understanding to the tachyon vortex 3.17 . Using $T^{2}=|x|^{2}$, one can show that $\mathcal{U}$ of 3.18 maps the origin $x=0$ to -1 in $U\left(2^{k}\right)$, and each point with $x^{2}=1$ to the identity in $U\left(2^{k}\right)$. Thus, 3.18 indeed defines a map from $\mathbf{S}^{2 k+1}$ to $U\left(2^{k}\right)$, and hence an element of $\pi_{2 k+1}\left(U\left(2^{k}\right)\right)$. This element in $\pi_{2 k+1}\left(U\left(2^{k}\right)\right)$ maps under 3.16 to the element in $\pi_{2 k}\left(\mathcal{V}_{1}\left(2^{k}\right)\right)$ that corresponds to the tachyon vortex 3.17 . (For details on this K-theory construction, see [30] .)

In terms of $\mathrm{K}$-theory, this proves that our tachyon condensate actually represents the generator of the relative $\mathrm{K}$-theory group $\mathrm{K}^{-1}\left(\mathbf{B}^{2 k+1}, \mathbf{S}^{2 k}\right)$, and our bound-state construction is precisely the analog of the ABS construction [25] , now mapping classes in $\mathrm{K}(Y)$ to classes in $\mathrm{K}^{-1}(X)$ for $Y$ of odd codimension in the spacetime manifold $X$ wrapped by the ustable 9-branes of Type IIA theory. ${ }^{9}$

This one-step construction of Type IIA D-branes as bound states of codimension $2 k+1$ in a system of unstable 9-branes suggests the following hierarchy of bound state constructions. Consider a supersymmetric $\mathrm{D} p$-brane in Type IIA or Type IIB theory. This D-brane can be constructed as a bound state (tachyon kink) in the worldvolume of an unstable $\mathrm{D}-(p+1)$ brane. Alternatively, it can be constructed $[1,2]$ as a bound state of a $(p+2)$-brane $\overline{(p+2)}$-brane pair. It can also be constructed as a bound state of two unstable $(p+3)$-branes, etc. This hierarchy of brane systems of increasing dimensions supports worldvolume gauge groups that form a natural

\footnotetext{
${ }^{9}$ The relative K-theory group $\mathrm{K}^{-1}\left(\mathbf{B}^{2 k+1}, \mathbf{S}^{2 k}\right)$ is defined as the group of equivalence classes of bundles with automorphisms $(E, \alpha)$ on $\mathbf{B}^{2 k+1}$, with $\alpha=1$ when restricted to the boundary $\mathbf{S}^{2 k}$ (see e.g. [22], section II.3.25.). In our string theory construction, $E$ is the Chan-Paton bundle, and as we have just seen, $\mathcal{U}$ of 3.18 has just the right properties to be identified with $\alpha$.
} 
hierarchy,

$U(1) \subset U(1) \times U(1) \subset U(2) \subset U(2) \times U(2) \subset U(4) \subset U(4) \times U(4) \ldots$

In this hierarchy, the bound state construction of $[1,2]$ in terms of pairs of stable branes alternates with the bound state construction in terms of ustable branes presented in this section. This procedure can be iterated until we reach the spacetime-filling dimension, thus ending up with a construction in terms of 9-branes where all spacetime symmetries are manifest.

\section{Codimension Three: The 't Hooft-Polyakov Monopole}

It is instructive to look more closely at the construction of bound states of codimension $2 k+1=3$. The gauge group suggested by $\mathrm{K}$-theory is $U(2)$, acting on a pair of unstable branes whose Chan-Paton bundle in 2 of $U(2)$ is identified with the two-dimensional spinor bundle $\mathcal{S}$ of the $S O(3)$ group of rotations in the transverse spacetime dimensions. The tachyon is a map from the two-dimensional spinor bundle $\mathcal{S}$ back to $\mathcal{S}$. Using the $\Gamma$-matrices $\sigma_{i}$ of $S O(3)$, the vortex of vorticity one can be written (up to an overall normalization factor) as

$$
T=\left(\begin{array}{cc}
x^{3} & x^{1}+i x^{2} \\
x^{1}-i x^{2} & -x^{3}
\end{array}\right) .
$$

The finite-energy condition ties 3.20 to a non-trivial gauge field, which takes the form

$$
A_{i}=f(|x|) \Gamma_{i j} x^{j}
$$

where $f(|x|)$ is a known convergence factor [34], and $\Gamma_{i j}$ is the antisymmetrized product of the $S O(3) \Gamma$-matrices $\sigma_{i}$ and $\sigma_{j}$. Up to the trivial lift from $S U(2)$ to $U(2)$ gauge theory, this is precisely the 't Hooft-Polyakov magnetic monopole in $3+1$ dimensions [34] ! In our construction, this monopole represents the supersymmetric stable D- $2 p$-brane of Type IIA theory as a bound state of two unstable $\mathrm{D}-(2 p+3)$-branes.

On the off-diagonal in 3.20 we recognize the tachyon condensate 2.4 that appeared in the construction $[1,2]$ of the D-2p-brane as a bound state of a $(2 p+2)$-brane $\overline{(2 p+2)}$-brane pair. Similarly, on the diagonal in 3.20 we find the tachyon kink 3.11 that corresponds to the construction of one $(2 p+2)$ brane and one $\overline{(2 p+2)}$-brane from a pair of $(2 p+3)$-branes. Thus, we can see that our one-step bound state construction can also be interpreted as a twostep construction, whereby we first prepare a $(2 p+2)$-brane $\overline{(2 p+2)}$-brane pair, which then forms a $2 p$-brane bound state. In this two-step construction, 
however, we lose some of the manifest symmetries of 3.20 by choosing an embedding of the $2 p$-brane worldvolume into a worldvolume of the $(2 p+2)$ brane $\overline{(2 p+2)}$-brane pair.

\section{Comments on Codimension One}

In codimension one, i.e. for $k=0$, the suggested value $2^{k}$ of the number of unstable Type IIA branes is one. Thus, there is no symmetry breaking of the $U(1)$ gauge symmetry, and we are left with a $U(1)$ gauge theory and a tachyon that can condense into either one of the two vacuum values, $\pm T_{0}$. This case was discussed at the beginning of this section, in the case of 8-branes and $\overline{8}$-branes as tachyon kinks on 9-brane worldvolumes. The symmetry restored at the core of the kink is the $\mathbf{Z}_{2}$ symmetry $T \rightarrow-T$.

We can now see from the K-theory perspective why this case of codimension-one bound states is rather degenerate. Indeed, the relation between homotopy groups of the vacuum manifold and those of the unitary groups, as given for $k \geq 1$ in 3.16 , becomes degenerate for $k=0$. The relevant homotopy group of the vacuum manifold for one 9 -brane is $\pi_{0}\left( \pm T_{0}\right)=\mathbf{Z}_{2}$, and there is not enough room for the anticipated conserved 8-brane charge that should be classified by $\mathbf{Z}$. Thus, each individual 8 -brane or $\overline{8}$-brane requires an extra 9-brane; the smallest 9-brane system that would accomodate the full $\mathrm{K}^{-1}\left(\mathbf{S}^{1}\right)=\mathbf{Z}$ group of 8-brane charges would contain an infinite number of 9-branes.

This also sheds some light on the two-step construction of the bound state. Consider a bound state of codimension $2 k+1$, as described in 3.17 - Choose $Y$ of codimension one, such that the worldvolume of our bound state lies in $Y$. Split the transverse dimensions as $2 k$ plus one, where the $2 k$ dimensions are within $Y$, and the last dimension is normal to $Y$. The tachyon vortex 3.17 can be written as

$$
T=\left(\begin{array}{cc}
x^{9} & \Gamma \cdot x \\
\Gamma^{\dagger} \cdot x & -x^{9}
\end{array}\right)
$$

where $x^{9}$ parametrizes the dimension transverse to $Y$, and $\Gamma$ are the $\Gamma$ matrices of $S O(2 k)$ along $Y .3 .22$ has a nice intuitive interpretation: the terms on the diagonal look precisely like $2^{k-1} 8$-branes and $2^{k-1} \overline{8}$-branes, and the off-diagonal terms correspond precisely to the bound state in the sense of [2] , which represents our brane as a bound state of codimension $2 k$ in a system of $2^{k-1} 8$-brane $\overline{8}$-brane pairs.

However, it is clear that such a two-step construction is artificial. Not only does it break manifest $S O(2 k+1)$ rotation invariance in the $2 k+1$ 
dimensions transverse to the worldvolume of our brane; it also artificially relates the non-degenerate case of $k \geq 1$ to the degenerate case of bound states of codimension one.

\section{Comparison to Bound States of Codimension One in Type I Theory}

Superficially, the construction presented above appears somewhat reminiscent of a construction $[1,2]$ in orientifolds of Type IIB theory, where certain stable non-supersymmetric $\mathrm{D} p$-branes carrying $\mathbf{Z}_{2}$ charges are realized as bound states of a number of pairs of stable $(p+1)$-branes and $\overline{(p+1)}$-branes. (Sen considers $p=0$ [1], while $p=8$ is discussed in [2] .) The following remark is intended to clarify the distinction between the two constructions.

We have seen that the tachyon condensate 3.17 on the worldvolume of unstable 9-branes of Type IIA theory represents a generator of the relative $\mathrm{K}$-theory group $\mathrm{K}^{-1}\left(\mathbf{B}^{2 k+1}, \mathbf{S}^{2 k}\right)=\mathbf{Z}$, and the bound state construction of a D-brane wrapping a submanifold $Y$ of codimension $2 k+1$ in spacetime $X$ represents a map from $\widetilde{\mathrm{K}}(Y)$ to $\mathrm{K}^{-1}(X)$. More precisely, this embedding of a lower-dimensional brane into the 9-brane system corresponds to the isomorphism

$$
\tilde{\mathrm{K}}(Y) \otimes \mathrm{K}^{-1}\left(\mathbf{B}^{2 k+1}, \mathbf{S}^{2 k}\right) \cong \mathrm{K}^{-1}\left(Y \times \mathbf{B}^{2 k+1}, Y \times \mathbf{S}^{2 k}\right),
$$

where $Y \times \mathbf{B}^{2 k+1}$ is a small neighborhood of $Y$ in spacetime. The tachyon 3.17 is a convenient representation of the generator of $\mathrm{K}^{-1}\left(\mathbf{B}^{2 k+1}, \mathbf{S}^{2 k}\right)$, and the isomorphism between $\widetilde{\mathrm{K}}(Y)$ and $\mathrm{K}^{-1}\left(Y \times \mathbf{B}^{2 k+1}, Y \times \mathbf{S}^{2 k}\right)$ is realized by the cup product with this generator.

In contrast, the tachyon kink used in [1] to describe the stable $\mathbf{Z}_{2}$-charged D0-brane of Type I theory as a bound state of a 1-brane 1-brane pair, represents the generator of $\operatorname{KO}\left(\mathbf{B}^{1}, \mathbf{S}^{0}\right)=\mathbf{Z}_{2}$. The cup product with this generator maps $\mathbf{Z}_{2}$ classes in KO-theory on $Y$ (representing the D0-brane carrying a $\mathbf{Z}_{2}$ charge $)$ to $\mathbf{Z}_{2}$ classes in $\mathrm{KO}\left(Y \times \mathbf{B}^{1}, Y \times \mathbf{S}^{\mathbf{0}}\right)$.

\subsection{Type $I^{\prime}$ Theory and $\mathrm{KR}^{-1}(X)$}

One can generalize the construction to the Type I' orientifold of Type IIA theory. The generalization is relatively straightforward, and we will be very brief.

Consider the orientifold of Type IIA theory on $\mathbf{R}^{10}$, with the orientifold group $\mathbf{Z}_{2}$ that changes the orientation of one spacetime dimension. This the- 
ory contains unstable spacetime-filling 9-branes, whose configurations up to creation and annihilation of elementary 9 -branes classify all possible $\mathrm{D}$-brane charges. In terms of $\mathrm{K}$-theory, this corresponds to a group called $\mathrm{KR}^{-1}(X)$ $[37,24,2]$, which can be defined as the group of equivalence classes of pairs $(E, \alpha)$, where $E$ is a bundle with an antilinear involution that commutes with the orientifold group, and $\alpha$ is an automorphism on $E$ that also preserves the orientifold group action. In terms of Type I' string theory, $E$ again corresponds to the Chan-Paton bundle on the worldvolume of the spacetimefilling 9-branes. At the orientifold planes, the gauge group is reduced from the unitary group to its orthogonal subgroup.

Each individual lower-dimensional D-brane can be represented as a bound state of a certain number of Type $\mathrm{I}^{\prime}$ 9-branes. The tachyon condensate is required to respect the orientifold $\mathbf{Z}_{2}$ symmetry, and therefore corresponds to what might be called a " $\mathbf{Z}_{2}$-equivariant monopole." (Similar equivariant solitons and instantons were studied in [12] .)

Far away from the orientifold planes, we can think of the spacetime manifold $X$ as being represented by a double cover $\tilde{X}$ of $X$ with the orientifold group mapping the two disconnected components of $\tilde{X}$ to each other. Using a standard result of $\mathrm{K}$-theory [37, 24]

$$
\mathrm{KR}^{-1}(\tilde{X}) \cong \mathrm{K}^{-1}(X),
$$

we can see that far away from the orientifold planes, we recover the Type IIA construction that occupied most of this section.

\section{K-Theory and Matrix Theory}

Consider $N$ D0-branes in Type IIA theory on $\mathbf{R}^{10}$, or a toroidal compactification thereof. (This restriction is mostly for simplicity; the construction can be straightforwardly extended to more complicated compactifications as well.) Using the general bound-state construction of section 3, each D0brane can be described as a bound state of sixteen unstable spacetime-filling 9-branes.

The worldvolume field theory of sixteen 9-branes contains a $U(16)$ gauge field, a tachyon $T$ in the adjoint of $U(16)$, and a pair of chiral fermions $\chi$ and $\chi^{\prime}$ of opposite chiralities, also in the adjoint of $U(16)$. In addition, we have the usual hierarchy of massive string states, and this system will couple to the Type IIA closed string sector.

We start with a configuration of sixteen 9-branes, in which the tachyon 
rolls down to a minimum $T_{0}$, with eight positive and eight negative eigenvalues $\pm T_{v}$. This condensate breaks $U(16)$ to $U(8) \times U(8)$, and the vacuum manifold is $\mathcal{V}=U(16) /(U(8) \times U(8))$. Since $\pi_{8}(\mathcal{V})=\mathbf{Z}$, the tachyon can develop a stable point-like vortex, whose form near the core can be described by

$$
T=\Gamma_{m} x^{m} .
$$

Here, again, $\Gamma_{m}$ are the $\Gamma$-matrices of $S O(9)$, the group of rotations in the dimensions transverse to the core of the vortex. This configuration carries vorticity one. In order to keep the energy of this localized object finite, the long-range gauge field will also be non-trivial, and will in fact give rise to a non-zero "magnetic charge" of the object. (More precisely, the corresponding 4 -th Chern class as measured by the gauge field on the 8-sphere surrounding the core of the vortex will be equal to one.)

The sixteen 9-branes are in the $\mathbf{1 6}$ of the gauge group $U(16)$. In the background of the generalized magnetic monopole/vortex representing the D0-brane, this $\mathbf{1 6}$ is identified with the spinor $\mathbf{1 6}$ of the $S O(9)$ spacetime rotation symmetry in the dimensions transverse to the worldvolume of the D0-brane. This represents a higher-dimensional generalization of the wellknown phenomenon in three space dimensions, where the background of the $S U(2)$ 't Hooft-Polyakov monopole identifies the $\mathbf{3}$ of the gauge group with the 3 of the space rotation group $S O(3)$.

A scaling argument [36] clearly suggests that the configuration will lower its energy by shrinking its core, and our description in terms of low-energy field theory on the 9-brane worldvolume will cease to be adequate. However, purely on topological grounds, one will be left with a stable soliton in string theory. The K-theory origin of this construction indicates that the soliton carries one unit of D0-brane charge, and therefore represents a D0-brane as a bound state of sixteen unstable 9-branes.

So far we have found the bound state that describes one D0-brane. Imagine now that we are interested in a system of $N$ D0-branes. There is no reason why we should use a new set of sixteen 9-branes for each individual D0-brane. (In terms of K-theory, the trivial topology of the D0-brane worldlines in flat spacetime does not require "stabilization" of the configuration by adding extra 9-branes.) Thus, in order to describe $N$ D0-branes, we do not have to add sixteen extra 9-branes each time we add a D0-brane - they can all be represented as bound states in a fixed system of sixteen spacetime-filling 9-branes.

Since we can construct a multi-D0-brane state using just sixteen Type IIA 9-branes, we can follow this 9-brane configuration as we take the scaling 
limit [19],[38]-[40] that defines Matrix theory. This suggests the intriguing possibility that we can formulate Matrix theory as a theory of stable solitons on the space-time filling worldvolume of sixteen unstable 9-branes! We can also add higher-dimensional D-branes to the system of D0-branes on $\mathbf{R}^{10}$, as vortices of codimension $2 k+1$ in the 9-brane worldvolume. These vortices are stable, since

$$
\pi_{2 k}(U(16) /(U(8) \times U(8)))=\mathbf{Z}
$$

(while all odd homotopy groups vanish). They are naturally formed as bound states of a number of 9-branes that is typically smaller than sixteen (in fact, the results of section 3 suggest that the number of 9-branes involved in a bound state of codimension $2 k+1$ is $2^{k}$ ), while the rest of the sixteen 9 branes are spectators in this construction. We can also use the bound state construction to study the full system of stable branes of various dimensionalities on a general spacetime manifold $X$. However, due to the non-trivial topology, this general case may require that extra 9-branes be added to the sixteen 9-branes that we have used in the description of D0-branes. The general construction would require the full $\mathrm{K}$-theory construction as discussed in the case of Type IIB theory in [2] . The "stabilization" by addition of 9-branes can be avoided for the system of $N$ D0-branes in flat spacetime, due to the trivial topology of the D0-brane worldlines.

This possible reinterpretation of Matrix theory in terms of vortices in a gauge theory with fixed gauge group is intriguing, since it allows us to change the number od D0-branes in the system without changing the rank of the gauge group. In Matrix theory, we would like to understand how systems with different values of $N$ are related to each other, possibly via some RG-like relation. This problem is notoriously difficult in the conventional formulation of Matrix theory, as it requires relating theories with gauge groups of different ranks. In contrast, the $\mathrm{K}$-theory construction of D0branes as magnetic vortices keeps the gauge group fixed for arbitrary values of $N$.

The non-supersymmetric $U(16)$ gauge theory on the worldvolume of sixteen 9-branes is defined through its embedding into the supersymmetric Type IIA string theory. In particular, it is unclear whether there are any useful limits in which supergravity decouples and leaves behind a system defined purely in terms of 9-brane degrees of freedom. Nevertheless, one might expect that at least for compactifications down to four dimensions or lower, interesting decoupling limits might exist, whereby D0-branes appear as magnetic vortices in a decoupled non-supersymmetric gauge theory.

It would be interesting to see directly in the bound-state construction how the system of $N$ D0-branes effectively decompactifies the eleventh dimension 
of Matrix theory [41, 42], and forms a "bubble" of the eleven-dimensional spacetime. Recall from section 2.2 that $\mathrm{K}^{-1}(X)$ is usually defined by starting with the K-theory of $X \times \mathbf{S}^{1}$, and imposing an extra condition that restricts the Type IIA D-brane charges to a subgroup $\mathrm{K}^{-1}(X) \subset \tilde{\mathrm{K}}\left(X \times \mathbf{S}^{1}\right)$. In other words, one can define $\mathrm{K}^{-1}(X)$ as K-theory of an eleven-dimensional extension of $X$, which is however not $X \times \mathbf{S}^{1}$ but rather $S^{\prime}(X)$, defined as $X \times$ $I$ with each boundary component "pinched" into a point. The replacement of $X \times \mathbf{S}^{1}$ by $S^{\prime}(X)$ is necessary to eliminate classes in $\widetilde{\mathrm{K}}\left(X \times \mathbf{S}^{1}\right)$ that are not in $\mathrm{K}^{-1}(X)$. For example, the one-point compactification of the tendimensional spacetime $\mathbf{R}^{10}$ is $\mathbf{S}^{10}$, but its suspension $S^{\prime}\left(\mathbf{R}^{10}\right)$ is an elevensphere. In combination with Matrix theory, the string theory construction of $\mathrm{K}^{-1}(X)$ in terms of bound states in 9-branes may perhaps alleviate some of the mystery related to the "M-theory" definition of $\mathrm{K}^{-1}(X)$ in terms of $\widetilde{\mathrm{K}}\left(X \times \mathbf{S}^{1}\right)$.

The construction of multi-D0-brane systems from a fixed system of 9branes, relevant to Matrix theory, has an analog in the case of D-strings of Type I theory [2] . It was pointed out in [2] that the Fock space of perturbative heterotic string theory should be contained in the system of eight 9-brane $\overline{9}$-brane pairs. Thus, the construction of [2] should have similar implications in heterotic Matrix string theory [43] .

\section{Comparison to Holographic Field Theory}

There is a number of intriguing similarities between this K-theory-inspired construction of a system of D0-branes in terms of vortices in a $U(16)$ gauge theory on the worldvolume of unstable 9-branes of Type IIA theory, and the ideas of holographic field theory suggested in the context of non-perturbative M-theory in [20] . Whether they are indicative of some closer relation remains to be seen.

Here is a list of some of them:

(1) In K-theory, D0-branes appear as vortices (or generalized magnetic monopoles) in a gauge theory with fixed rank. Similarly, in holographic field theory, the partons (to be compared to D0-branes of Matrix theory) appear as vortices in a fixed-rank gauge group. Thus, in both cases, the limit to be compared to matrix theory requires one to look at multi-particle systems of vortices in a fixed-rank gauge group. D0-branes would appear as solitonic excitations in a medium not dissimilar to some condensed matter systems.

(2) The gauge group of holographic field theory is

$$
\mathcal{G}=O S p(1 \mid 32) \times O S p(1 \mid 32) .
$$


For many topological purposes, this group can be considered equivalent to its maximal compact subgroup. The maximal compact subgroup of this non-compact version of $O S p(1 \mid 32)$ is, in fact, $U(16)$. When extended beyond ten dimensions, the natural hierarchy of gauge groups 3.19 suggests $U(16) \times U(16)$ as the group relevant to eleven dimensions. $U(16) \times U(16)$ is the maximal compact subgroup of the gauge group 4.3 of holographic field theory.

(3) The gauge group $\mathcal{G}$ can be interpreted as the minimal extension of AdS group in eleven dimensions compatible with supersymmetry and parity invariance. Perhaps more interestingly, it can also be viewed as a nonchiral Lorentz group in $(10,2)$ dimensions [20] . This leads to a convenient representation of the bosonic subgroup $S p(32, \mathbf{R}) \times S p(32, \mathbf{R})$ in terms of $64 \times 64 \Gamma$-matrices acting on the spinor bundle $\mathcal{S}=\mathcal{S}_{+} \oplus \mathcal{S}_{-}$whose sections are 64-component non-chiral spinors in $(10,2)$ dimensions. Each $S p(32, \mathbf{R})$ acts on one of the two factors in $\mathcal{S}_{+} \oplus \mathcal{S}_{-}$. This is again very reminiscent of structures appearing in K-theory.

(4) The previous point can be extended even further. The construction that embeds lower-dimensional branes as bound states into systems of higher-dimensional branes is of course a known K-theory construction [25] . The precise mathematical form of this map is realized via the Euler class of the normal bundle of the embedding of the lower-dimensional brane into the worldvolume of the higher-dimensional branes (see, e.g., [22] , sect. IV.1). The Lagrangian of holographic field theory [20] is intimately related to the Euler class. More exactly, the Lagrangian can be interpreted as the Euler class of the tangent space to the twelve-dimensional manifold that has the eleven-dimensional spacetime as its boundary: the exterior derivative of the Chern-Simons Lagrangian of [20] is a supersymmetrization of the Euler density in $(10,2)$ dimensions.

It is a pleasure to thank Oren Bergman, Eric Gimon, Djordje Minic, Michael Peskin, John Preskill, John Schwarz, Steve Shenker, Eva Silverstein, Lenny Susskind and Edward Witten for valuable discussions. I wish to thank the Stanford Institute of Theoretical Physics for hospitality during some parts of this work. This work has been supported by Sherman Fairchild Prize Fellowship and by DOE Grant DE-FG03-92-ER 40701. 


\section{References}

[1] A. Sen, "Stable Non-BPS States in String Theory," J. High Energy Phys. 06 (1998) 007, hep-th/9803194; "Stable Non-BPS Bound States of BPS D-Branes," J. High Energy Phys. 08 (1998) 010, hepth/9805019; "Tachyon Condensation on the Brane Antibrane System," J. High Energy Phys. 08 (1998) 012, hep-th/9805170; "SO(32) Spinors of Type I and Other Solitons on Brane-Antibrane Pair," J. High Energy Phys. 09 (1998) 023, hep-th/9808141; "Type I D-Particle and its Interactions," J. High Energy Phys. 10 (1998) 021, hep-th/9809111.

[2] E. Witten, " $D$-Branes and K-Theory," hep-th/9810188.

[3] T. Banks and L. Susskind, "Brane - Antibrane Forces," hepth/9511194.

[4] G. Lifschytz, "Comparing D-Branes to Black-branes," Phys. Lett. B388 (1996) 720 , hep-th/9604156.

[5] V. Periwal, "Antibranes and Crossing Symmetry," hep-th/9612215.

[6] M.B. Green and M. Gutperle, "Light-Cone Supersymmetry and DBranes," Nucl. Phys. B476 (1996) 484, hep-th/9604091.

[7] M. Srednicki, "IIB or not IIB," J. High Energy Phys. 08 (1998) 005, hep-th/9807138.

[8] O. Bergman and M.R. Gaberdiel, "Stable Non-BPS D-Particles," hepth/9806155

[9] E. Gava, K.S. Narain and M.H. Sarmadi, "On the Bound States of $p$ and ( $p+2)$-Branes," Nucl. Phys. B504 (1997) 214, hep-th/9704006.

[10] R. Minasian and G. Moore, " $K$-Theory and Ramond-Ramond Charge," J. High Energy Phys. 11 (1997) 002, hep-th/9710230.

[11] P. Hořava, "Orbifold Approach to Open String Theory," PhD. Thesis (Institute of Physics, Czech. Acad. Sci., Prague, 1991)

[12] P. Hořava, "Equivariant Topological Sigma Models," Nucl. Phys. B418 (1994) 571, hep-th/9309124.

[13] M. Bershadsky, V. Sadov and C. Vafa, "D-Branes and Topological Field Theories," Nucl. Phys. B463 (1996) 420, hep-th/9511222.

[14] A. Strominger, "Open P-Branes," Phys. Lett. B383 (1996) 44, hepth/9512059. 
[15] M.R. Douglas, "Branes within Branes," hep-th/9512077.

[16] M.R. Douglas and G. Moore, "D-Branes, Quivers, and ALE Instantons," hep-th/9603167.

[17] M.B. Green, J.A. Harvey and G. Moore, 'I-Brane Inflow and Anomalous Couplings on D-Branes," Class. Q. Grav. 14 (1997) 47, hep-th/9605033.

[18] Y.-K. E. Cheung and Z. Yin, "Anomalies, Branes, and Currents," Nucl. Phys. B517 (1998) 69, hep-th/9710206.

[19] T. Banks, W. Fischler, S.H. Shenker and L. Susskind, "M Theory as a Matrix Model," Phys. Rev. D55 (1997) 5112, hep-th/9610043.

[20] P. Hořava, "M-Theory as a Holographic Field Theory," hep-th/9712130, to appear in Phys. Rev. D.

[21] A. Sen, "BPS D-Branes on Non-supersymmetric Cycles," hepth/9812031.

[22] M. Karoubi, K-Theory. An Introduction (Springer, 1978).

[23] D. Husemoller, Fibre Bundles (1st ed., Mc Graw-Hill, 1966; 3rd ed., Springer, 1994).

[24] H. B. Lawson, Jr. and M.-L. Michelsohn, Spin Geometry (Princeton, 1989).

[25] M.F. Atiyah, R. Bott and A. Shapiro, "Clifford Modules," Topology 3 Suppl. 1 (1964) 3.

[26] M.F. Atiyah, K-Theory (Benjamin, 1964).

[27] D. Quillen, "Superconnections and the Chern Character," Topology 24 (1985) 89

N. Berline, E. Getzler and M. Vergne, Heat Kernels and Dirac Operators (Springer, 1992).

[28] N. Marcus and A. Sagnotti, "Group Theory from 'Quarks' at the Ends of Strings," Phys. Lett. B188 (1987) 58.

[29] L.-F. Li, "Group Theory of the Spontaneously Broken Gauge Symmetries," Phys. Rev. D9 (1974) 1723.

[30] B.A. Dubrovin, A.T. Fomenko and S.P. Novikov, Modern Geometry Methods and Applications, pt. III (Springer, 1990), section III.25.1.

[31] J. Polchinski and Y. Cai, "Consistency of Open Superstring Theories," Nucl. Phys. B296 (1988) 91. 
[32] C.G. Callan, C. Lovelace, C.R. Nappi and S.A. Yost, "Adding Holes and Crosscaps to the Superstring," Nucl. Phys. B293 (1987) 83.

[33] P. Hořava, "Strings on World-Sheet Orbifolds," Nucl. Phys. B327 (1989) 461.

[34] G. 't Hooft, "Magnetic Monopoles in Unified Gauge Theories," Nucl. Phys. B79 (1974) 276

A.M. Polyakov, "Particle Spectrum in Quantum Field Theory," JETP Lett. 20 (1974) 194,

reprinted in C. Rebbi and G. Soliani, Solitons and Particles (World Scientific, 1984).

[35] S. Coleman, "Classical Lumps and Their Quantum Descendants," 1975 Erice lectures, reprinted in: S. Coleman, Aspects of Symmetry (Cambridge, 1985)

P. Goddard and D.I. Olive, "Magnetic Monopoles in Gauge Field Theories," Rep. Prog. Phys. 41 (1978) 1357

J. Preskill, "Vortices and Monopoles," 1985 Les Houches lectures, in: Architecture of Fundamental Interactions at Short Distances, eds. P. Ramond and R. Stora (Elsevier, 1987)

J.A. Harvey, "Magnetic Monopoles, Duality and Supersymmetry," hepth/9603086.

[36] S. Weinberg, The Quantum Theory of Fields, Vol. II (Cambridge, 1996), sect. 23.1.

[37] M.F. Atiyah, "K-Theory and Reality," Quart. J. Math. Oxford 17 (1966) 367; reprinted in [26] .

[38] M.R. Douglas, D. Kabat, P. Pouliot and S.H. Shenker, "D-Branes and Short Distances in String Theory," Nucl. Phys. B485 (1997) 85, hepth/9608024.

[39] A. Sen, "D0 Branes on $T^{n}$ and Matrix Theory," Adv. Theor. Math. Phys. 2 (1998) 51, hep-th/9709220.

[40] N. Seiberg, "Why is the Matrix Model Correct?," Phys. Rev. Lett. 79 (1997) 3577 , hep-th/9710009.

[41] K. Becker, M. Becker, J. Polchinski and A. Tseytlin, "Higher Order Graviton Scattering in M(atrix) Theory," Phys. Rev. D56 (1997) 3174, hep-th/9706072. 
[42] V. Balasubramanian, R. Gopakumar and F. Larsen, "Gauge Theory, Geometry and the Large N Limit," Nucl. Phys. $B 526$ (1998) 415, hepth/9712077.

[43] T. Banks and L. Motl, "Heterotic Strings from Matrices," J. High Energy Phys. 12 (1997) 004, hep-th/9703218

D. Lowe, "Heterotic Matrix String Theory," Phys. Lett. B403 (1997) 243, hep-th/9704041

S.-J. Rey, Heterotic M(atrix) Strings and Their Interactions," Nucl. Phys. B502 (1997) 170, hep-th/9704158

P. Hořava, "Matrix Theory and Heterotic Strings on Tori," Nucl. Phys. B505 (1997) 84. 